\title{
Esquemas disfuncionales tempranos y consumo de alcohol en estudiantes universitarios, según carrera profesional de una universidad pública de la ciudad de Lima
}

\author{
Early maladaptive schemes and alcohol consumption in university \\ students, according to professional career of a public university of the city \\ of Lima
}

\author{
Rosa E. Huerta R. ${ }^{1}$ \\ Manuel Miljanovich ${ }^{2}$ \\ Jaime Aliaga ${ }^{3}$ \\ Emma Campos ${ }^{4}$
}

\author{
Natalia Ramírez 5 \\ Eliana Delgado ${ }^{6}$ \\ Jessica Ramos ${ }^{7}$ \\ Lourdes Murillo ${ }^{8}$
}

Universidad Nacional Mayor de San Marcos

\author{
Recibido: $29-08-17$
}

Aceptado: $21-11-17$

\begin{abstract}
Resumen
En el estudio se sometió al análisis las variables esquemas cognitivos disfuncionales tempranos y consumo de alcohol según carrera profesional, ciencias de la salud e ingeniería en una muestra de 510 estudiantes universitarios, a quienes se les administró una ficha de datos sociodemográficos, el Cuestionario de Esquemas de Young integrado por 18 esquemas y el Cuestionario de Identificación de los Trastornos debidos al Consumo de Alcohol (AUDIT). Se encontró que existe una correlación significativa y positiva entre los esquemas disfuncionales tempranos, privación emocional, grandiosidad insuficiente autocontrol y reconocimiento con el consumo de alcohol, En ciencias de la salud en el grupo de consumo de riesgo predomina el esquema autosacrificio y en ingeniería los esquemas dependencia /incompetencia y subyugación. Los estudiantes con consumo de riesgo más alto son de ingeniería (31) en comparación con los de ciencias de la salud que fue menor (7). Los estudiantes de ingeniería presentan mayor consumo de alcohol. Resultados que son graves pues el consumo de alcohol se asocia con esquemas cognitivos caracterizados por la sobreestimación al daño, baja tolerancia a la frustración y alta necesidad de aprobación social
\end{abstract}

Palabras claves: Esquemas disfuncionales tempranos; consumo de alcohol; carrera profesional y estudiantes universitarios.

Universidad Nacional Mayor de San Marcos. * correspondencia rhuertar@unmsm.edu.pe

Universidad Nacional Mayor de San Marcos. mmiljanovichc@unmsm.edu.pe

Universidad Nacional Mayor de San Marcos. jaliagat@unmsm.edu.pe

Universidad Nacional Mayor de San Marcos. ecamposp@unmsm.edu.pe

Universidad Nacional Mayor de San Marcos. nramirezs@unmsm.edu.pe

Universidad Nacional Mayor de San Marcos. edelgadoc@unmsm.edu.pe

Universidad Nacional Mayor de San Marcos. jessicaramosreyes@gmail.com

Universidad Nacional Mayor de San Marcos. lourdesmurillor@hotmail.com

(C) Los autores. Este artículo es publicado por la Revista de Investigación en Psicología de la Facultad de Psicología, Universidad Nacional Mayor de San Marcos. Este es un artículo de acceso abierto, distribuido bajo los términos de la licencia Creative Commons Atribucion - No Comercia_Compartir Igual 4.0 Internacional. (http://creativecommons.org/licenses/by-nc-sa/4.0/) que permite el uso no comercial, distribución y reproducción en cualquier medio, siempre que la obra original sea debidamente citada. 


\begin{abstract}
The study has been subjected to the analysis of the variables early maladaptive schemas and alcohol consumption by career, health sciences and engineering in a sample of 510 university students, who were given a socio-demographic data sheet, the Young Schema Questionnaire composed of 18 schemes and the Alcohol Use Disorders Identification Test (AUDIT).It was found that there is a significant and positive correlation between early maladaptive schemas, emotional deprivation, insufficient self-control and recognition with alcohol consumption. In health sciences, the risk-taking group predominates the autosacrifice scheme and in engineering the schemes of Dependence / incompetence and subjugation. The students with higher risk consumption are engineering (31) compared to those in health sciences which was lower (7). Engineering students have higher alcohol consumption. Results that are serious because the alcohol consumption is associated with cognitive schemes characterized by the overestimation to the damage, low tolerance to the frustration and high necessity of social approval.

Keywords: Early maladaptive schemes; alcohol consumption; career and college students.
\end{abstract}

El consumo de alcohol en jóvenes es un fenómeno complicado de determinar debido a múltiples factores asociados, entre los que se encuentran creencias, imaginarios sociales, estilos de vida, estados afectivos, factores motivacionales, procesos conductuales de ingesta, edad, antecedentes familiares de consumo, presión de grupo y en general el modelo disfuncional de consumo de compañeros y amigos; con consecuencias nefastas a nivel intelectual, problemas de rendimiento académico, familiares, depresión, entre alguno de ellos, (Cooper, 1999, citado en Andrade, Yepes y Sabogal; 2013).

En el Perú el consumo de alcohol es una problemática social, cuyo incremento en los últimos años en la población joven es innegable, agravado por un inicio en una edad cada vez más temprana, 13 años (DEVIDA, 2013). El alcohol es la sustancia psicoactiva de mayor uso en todas las edades, con la desventaja de ser aceptada y consentida socialmente (84\%, DEVIDA, 2014). Afectando áreas básicas del desarrollo de la persona, como es su salud tanto física como mental. El alcohol es una sustancia psicoactiva que forma parte del grupo de depresores del sistema nervioso central, lo que conlleva a observar en quienes la consumen mengua del estado de conciencia, de su capacidad de responder a la estimulación sensorial, de su expresión espontánea, del trabajo físico y un bajo rendimiento cognitivo.

En lo que se refiere a la ingesta de alcohol en estudiantes universitarios, el II Estudio Epidemiológico Andino sobre Consumo de Drogas en la Población Universitaria Informe Perú, 2012 (Comunidad Andina, 2013) encontró una prevalencia de consumo reciente de 71.7 y una incidencia de 70.57. Asimismo advierte que el $10.8 \%$, es decir 1 de cada 9 estudiantes que indican haber consumido alcohol en el último año, presentan indicadores compatibles con la dependencia alcohólica, destacando los varones universitarios, $14.5 \%$, respecto de las mujeres, $6.6 \%$, tasa que confirma que el abuso de alcohol y otras drogas ha dejado de ser "exclusividad" del género masculino. La edad media de inicio del consumo de 
alcohol es 16 años, con la diferencia de 1 año entre varones y mujeres, 15.7 y 16.6 años, correspondientemente (Comunidad Andina, 2013).

De acuerdo con Londoño, García, Valencia y Vinaccia (2005), la cultura universitaria establecida por jóvenes los suministra de un conjunto de aseveraciones positivas acerca del consumo del alcohol y sus alcances, mismas que son asumidas como afirmaciones dentro de los esquemas cognitivos del joven actuando como modificadoras del comportamiento de consumo.

Los esquemas cognitivos intervienen en el modo de conducirse, de pensar y decidir sobre hechos que se manifiestan en nuestro ciclo vital, asimismo influyen en la manera como la persona toma en cuenta a los demás y en la modo de vincularse con las mismas (Hederich \& Camargo, 2001; citado en Gantiva, Bello, Vanegas, y Sastoque, (2010). Los esquemas se precisan como la percepción, la organización y el proceso de la información, que se presentan en variaciones en las estrategias que siguen los sujetos en el momento que realizan una tarea cognitiva.

Young (1999) plantea sus propuestas teóricas de esquemas disfuncionales tempranos que se describen como una estructura muy estable y duradera que tienen lugar en la infancia, se construyen durante la vida de la persona, son excesivamente disfuncionales los que pasan a ser referentes para el procesamiento de experiencias posteriores las cuales emergen o son activados cuando son disparados por eventos de todos los días que son relevantes para los esquemas, (Young, 1999).

Gantiva, et al (2010) identificaron una asociación entre el consumo de alcohol y los esquemas disfuncionales destacando los de abandono, autocontrol insuficiente, desconfianza, vulnerabilidad, derecho/grandiosidad, autosacrificio y entrampamiento. La ingesta de alcohol se asocia con estilos cognitivos determinados por sobre estimación al sufrimiento, poca conformidad a la frustración y gran exigencia por ser aprobado socialmente.

Chattás y Góngora (2008) señala que los sujetos con adicciones poseen esquemas disfuncionales tempranos más severos que los sujetos sin adicción, destacándose esquemas de Privación Emocional, Abandono /Inestabilidad; Desconfianza / Abuso y Dependencia / Incompetencia

Desde esta perspectiva los esquemas cognitivos influirán de manera concreta en la forma en que los sujetos perciben el mundo y, como consecuencia, esta distorsión cognitiva llevará al sujeto a padecer desadaptación o disfuncionalidad ante situaciones que no tendrían que ser así, ya que no se corresponden con la realidad (Young, 1999, citado en Castrillón et al., 2005). Estos se refieren a creencias básicas que predisponen al individuo a una vulnerabilidad psicopatológica (Sanchez y Sanchez, 1999; citado en Chattás y Góngora, 2008). Una de las consecuencias que describen durante la edad adulta es la toxicomanía. 
Los esquemas disfuncionales tempranos intervienen en el consumo de alcohol. Así, McGinn y Young (1996) refirieron que los esquemas cognitivos se manifiestan por los ambientes estresantes que son significativos a cada esquema particular. Cuando estos se accionan, los esquemas dan lugar a emociones enormemente fuertes en el individuo, conduciéndolo directa o indirectamente a una sucesión de dificultades psicológicas como depresión, emociones de soledad, relaciones maltratadoras, adicciones al alcohol y drogas.

Para el presente estudio se planteó como objetivos, analizar la relación entre los esquemas disfuncionales tempranos y el consumo de alcohol en estudiantes universitarios de quinto ciclo, según carrera profesional (Ciencias de la Salud e Ingenierías), de una universidad pública de la ciudad de Lima; así mismo comparar las diferencias entre los esquemas disfuncionales tempranos y el consumo de alcohol en estudiantes universitarios de ciencias de la salud e ingenierías. Otro objetivo ha sido adaptar el Cuestionario de Esquemas Cognitivos de Young, (YSQ-S3).

\section{MÉTODO}

El presente estudio es de tipo sustantivo, que sistematiza procedimientos dentro del método correlacional y adicionalmente de la contrastación que nos permitió conocer tanto la intensidad y dirección de la relación entre la los esquemas disfuncionales tempranos y el consumo de alcohol en estudiantes universitarios de quinto ciclo, según carrera profesional (Ciencias de la Salud e Ingenierías), de una universidad pública de la ciudad de Lima. El diseño fue descriptivo correlacional, transversal, los datos fueron recogidos en un solo momento (Hernández, Fernández y Baptista, 2010).

\section{Muestra}

El universo de investigación está conformado por estudiantes universitarios de quinto ciclo que asisten a una universidad pública de la ciudad de Lima. El tamaño de la muestra se determinó aplicándose un muestreo no probabilístico, intencional.

La muestra quedo constituida por 510 estudiantes de ambos sexos que clasificaron según edad y carrera profesional como se describe a continuación:

Se observa que las edades fluctuaron entre 18 y 51 años predominando la edad de 21 a 23 años tanto en el grupo de estudiantes universitarios de la carrera de ciencias de la salud (298) como en la de ingenierías (212), en cuanto al género fueron 197 mujeres y 313 hombres.

\section{Variables de Estudio}

Las variables de estudio corresponden a los indicadores del Cuestionario de Esquemas de Young (YSQ- S3) y al Cuestionario de Identificación de los Trastornos 
debidos al Consumo de Alcohol (AUDIT) los que fueron aplicadas a los dos grupos de estudiantes universitarios del quinto ciclo de las carreras de ciencias de la salud e ingenierías, por lo cual se puede establecer la siguiente asignación de variables:

- Variable Independiente: Estudiantes universitarios de quinto ciclo de las carreras de ciencias de la salud e ingenierías que asisten a una universidad pública de la ciudad de Lima.

- Variable Dependiente: Puntajes de las pruebas aplicadas

\section{Técnicas e Instrumentos de recolección de datos.}

Con el fin de recoger de datos se determinaron las Facultades y Escuelas de una Universidad pública de la ciudad de Lima. La aplicación de los instrumentos se realizó de manera colectiva.

Se utilizó la técnica de la encuesta y dos instrumentos. Las Escalas que a continuación se describen previamente se revisaron la traducción (en el caso del Cuestionario YSQ-S3) y fueron adaptadas lingüísticamente; además el investigador elaboró una ficha para identificar los datos sociodemográficos de los estudiantes.

\section{Instrumentos:}

- Ficha de datos sociodemográficos: Elaborada por el investigador. Aplicada de manera colectiva. Permite recoger características de los sujetos estudiados como, lugar de procedencia, edad, sexo, semestre de estudios, con quién vive, así como el estado civil de los padres y si tiene actividad laboral.

- Cuestionario de Esquemas de Young (YSQ- S3) de Jeffrey Young. Es un instrumento de indagación aplicable a sujetos a partir de los 16 años. Consta de 90 reactivos, cada uno de ellos está estructurado en una escala tipo Likert por seis enunciados, de los cuales el sujeto tiene que seleccionar el que mejor describa su forma de evaluar una situación. Evalúa los esquemas disfuncionales tempranos o denominado también esquemas maladaptativos tempranos. Mide 18 factores agrupados en cinco dimensiones.

Se ha revisado la traducción y se ha realizado la adaptación lingüística, procediéndose a obtener la validez de constructo y la confiabilidad llegando según el alfa de Cronbach $r=0.97$. Castrillon, et al (2005), elabora una versión de este cuestionario y obtiene un alfa de Cronbach de 0.95 .

- Cuestionario de Identificación de los Trastornos debidos al Consumo de Alcohol-AUDIT: elaborado en 1989 y revisada por Babor, Higgins, 
Saunders y Monteiro (2001) para la Organización Mundial de la Salud (OMS) como un método simple de screning al consumo de alcohol y apoyo en la evaluación breve, para identificar a las personas con un patrón de consumo de riesgo, consumo perjudicial o consumo con síntomas de dependencia de alcohol. Consta de 10 preguntas, Aplicable a adolescentes mayores de 15 años y adultos. El punto de corte para consumo es 8 . Un estudio de fiabilidad test-retest indico una alta fiabilidad $(r=.86)$ en una muestra compuesta por bebedores sin consumo de riesgo, sujetos con abuso de cocaína y alcohólicos. Para el presente estudio se encontró una confiabilidad según el Alpha de Cronbach de 0.805.

\section{RESULTADOS}

1. Relación entre los esquemas disfuncionales tempranos y los niveles de consumo de alcohol en estudiantes universitarios de quinto ciclo, según carrera profesional (Ciencias de la Salud e Ingenierías) de una universidad pública de la ciudad de Lima. Los datos obtenidos de ambas variables se correlacionaron con el coeficiente Rho de Spearman encontrando relaciones significativas y positivas entre las dimensiones de los esquemas disfuncionales tempranos con los niveles de consumo de alcohol:

Al analizar los datos en la muestra total, se encuentra que existe correlación estadísticamente significativa positiva, aunque baja (menor a 0.20 ) entre los esquemas de privación emocional, grandiosidad/autorización, insuficiente autocontrol y búsqueda de aprobación/reconocimiento con el consumo de alcohol. En ese sentido, los evaluados que presentan en mayor medida los esquemas de privación emocional, grandiosidad/autorización, insuficiente autocontrol y búsqueda de aprobación/reconocimiento, tienden a presentar mayor consumo de alcohol.

En relación a estudiantes de ciencias de la salud, existe correlación estadísticamente significativa positiva y baja (menor a 0.20) entre el esquema castigo con el consumo de alcohol. En ese sentido, los estudiantes de ciencias de la salud que presentan en mayor medida el esquema de castigo, tienden a presentar mayor consumo de alcohol. Es decir los universitarios consideran que las personas deben ser castigadas por sus errores, lo que conlleva a impaciencia y a no tolerar sus propios errores ni la de los demás

En el caso de los estudiantes de las carreras de ingeniería, existe correlación estadísticamente significativa positiva y baja (menor a 0.20 ) entre los esquemas de privación emocional, desconfianza/abuso y búsqueda de aprobación/ reconocimiento con el consumo de alcohol. En ese sentido, los estudiantes de carreras de ingeniería que presentan en mayor medida estos esquemas, tienden a presentar mayor consumo de alcohol. Ellos presentan la expectativa de que los 
demás los humillarán, que los engañarán o se aprovecharán de él, que conllevará a que no reciban afecto por lo que tratan de ganarse el afecto de los demás a costa de su propia identidad. Como se puede observar en la Tabla 1.

Tabla 1

Correlación entre esquemas disfuncionales tempranos y consumo de alcohol

\begin{tabular}{|c|c|c|c|c|}
\hline & & \multicolumn{3}{|c|}{ Consumo de alcohol } \\
\hline & & Total & Ciencias de la salud & Ingenierías \\
\hline \multirow{2}{*}{ Privación Emocional } & Rho & $.096^{*}$ & .041 & $.162^{*}$ \\
\hline & $\mathrm{p}$ & .030 & .476 & .018 \\
\hline \multirow{2}{*}{ Abandono/ inestabilidad } & Rho & .058 & .037 & .109 \\
\hline & $\mathrm{p}$ & .188 & .529 & .112 \\
\hline \multirow{2}{*}{ Desconfianza/abuso } & Rho & .080 & .021 & $.146^{*}$ \\
\hline & $\mathrm{p}$ & .069 & .712 & .034 \\
\hline \multirow{2}{*}{ Aislamiento Social/Alienación } & Rho & .010 & .000 & .031 \\
\hline & $\mathrm{p}$ & .819 & .994 & 649 \\
\hline \multirow{2}{*}{ Imperfección/ vergüenza } & Rho & .026 & .020 & .033 \\
\hline & $\mathrm{p}$ & .560 & .734 & .631 \\
\hline \multirow{2}{*}{ Fracaso } & Rho & -.018 & -.022 & .019 \\
\hline & $\mathrm{p}$ & .681 & .702 & .788 \\
\hline \multirow{2}{*}{ Dependencia/ incompetencia } & Rho & .026 & -.008 & .092 \\
\hline & $\mathrm{p}$ & .557 & .893 & .182 \\
\hline \multirow{2}{*}{ Vulnerabilidad al peligro o a la enfermedad } & Rho & .024 & -.010 & .045 \\
\hline & $\mathrm{p}$ & .594 & .868 & .516 \\
\hline \multirow{2}{*}{ Apego confuso/ Yo inmaduro } & Rho & .011 & -.082 & .111 \\
\hline & $\mathrm{p}$ & .805 & .160 & .108 \\
\hline \multirow{2}{*}{ Subyugación } & Rho & .035 & -.019 & .112 \\
\hline & $\mathrm{p}$ & .425 & .748 & .105 \\
\hline \multirow{2}{*}{ Autosacrificio } & Rho & -.034 & -.096 & .049 \\
\hline & $\mathrm{p}$ & .442 & .099 & .478 \\
\hline \multirow{2}{*}{ Inhibición emocional } & Rho & .039 & -.014 & .045 \\
\hline & $\mathrm{p}$ & .386 & .807 & .518 \\
\hline \multirow{2}{*}{ Normas inalcanzables / hipercriticismo } & Rho & -.009 & -.010 & -.020 \\
\hline & $\mathrm{p}$ & .836 & .861 & .773 \\
\hline \multirow{2}{*}{ Grandiosidad/ autorización } & Rho & $.125^{* *}$ & .072 & .129 \\
\hline & $\mathrm{p}$ & .005 & .212 & .060 \\
\hline \multirow{2}{*}{ Insuficiente autocontrol } & Rho & $.091^{*}$ & .065 & .068 \\
\hline & $\mathrm{p}$ & .039 & .260 & .327 \\
\hline \multirow{2}{*}{ Búsqueda de aprobación /reconocimiento } & Rho & $.139^{* *}$ & .076 & $.185^{\text {** }}$ \\
\hline & $\mathrm{p}$ & .002 & .189 & .007 \\
\hline \multirow{2}{*}{ Negatividad/ Pesimismo } & Rho & .085 & .093 & .020 \\
\hline & $\mathrm{p}$ & .055 & .109 & .769 \\
\hline \multirow{3}{*}{ Castigo } & Rho & .080 & $.116^{*}$ & .064 \\
\hline & $\mathrm{p}$ & .072 & .046 & .356 \\
\hline & $\mathrm{N}$ & 510 & 298 & 212 \\
\hline
\end{tabular}

*. La correlación es significativa al nivel 0.05 (bilateral).

**. La correlación es significativa al nivel 0.01 (bilateral). 
2. Diferencias entre los esquemas disfuncionales tempranos con los niveles de consumo de alcohol en estudiantes universitarios de quinto ciclo, según Carrera profesional (Ciencias de la Salud e Ingenierías) de una universidad pública de la ciudad de Lima. El presente análisis efectuado en estudiantes universitarios con riesgo y sin riesgo en el consumo de alcohol de una universidad pública de la ciudad de Lima, para lo cual se ha contrastado ambos grupos, utilizando el análisis U de Mann Whitney, con el objeto de conocer si difieren en función a las dimensiones en sus esquemas cognitivos disfuncionales tempranos según la presencia y no de consumo de riesgo de alcohol encontrando:

a. Al analizar la muestra total, contrastando el total de estudiantes universitarios de las carrera de ciencias de la salud e ingenierías divididos en consumo de riesgo y sin riesgo se observan diferencias en cuanto a los esquemas disfuncionales tempranos y sus dominios según nivel de consumo, apreciándose mayores rangos medios en el grupo con riesgo que en los sin riesgo de consumo de alcohol, como se aprecia en la Tabla 2.

Tabla 2

Comparación de los esquemas disfuncionales y sus dominios según nivel de consumo de alcohol en la muestra total.

\begin{tabular}{lcccc}
\hline \multicolumn{1}{c}{ Esquema } & \multicolumn{4}{c}{ Muestra total } \\
& $\begin{array}{c}\text { U de Mann- } \\
\text { Whitney }\end{array}$ & $\begin{array}{c}\text { Rango promedio } \\
\text { para sin riesgo de } \\
\text { consumo } \\
\text { (N= 472) }\end{array}$ & $\begin{array}{c}\text { Rango promedio } \\
\text { para con riesgo de } \\
\text { consumo } \\
\text { (N = 38) }\end{array}$ & p \\
\hline Privación Emocional & 7459.50 & 252.30 & 295.20 & .083 \\
Abandono/ inestabilidad & 7432.00 & 252.25 & 295.92 & .078 \\
Desconfianza/abuso & 7104.00 & 251.55 & 304.55 & $\mathbf{. 0 3 3}$ \\
Aislamiento Social/Alienación & 8500.50 & 254.51 & 267.80 & .592 \\
Imperfección/ vergüenza & 8243.50 & 253.97 & 274.57 & .405 \\
Fracaso & 7766.00 & 252.95 & 287.13 & .167 \\
Dependencia/ incompetencia & 6898.00 & 251.11 & 309.97 & $\mathbf{. 0 1 7}$ \\
Vulnerabilidad al peligro o a la & 7230.00 & 251.82 & 301.24 & $\mathbf{. 0 4 6}$ \\
enfermedad & 7184.50 & 251.72 & 302.43 & $\mathbf{. 0 4 1}$ \\
Apego confuso/ Yo inmaduro & 6945.50 & 251.22 & 308.72 & .020 \\
Subyugación & 6992.00 & 251.31 & 307.50 & $\mathbf{. 0 2 3}$ \\
Autosacrificio & 8119.00 & 253.70 & 277.84 & .330 \\
Inhibición emocional & 8506.00 & 256.48 & 243.34 & .596 \\
Normas inalcanzables / & 7875.00 & 253.18 & 284.26 & .210 \\
hipercriticismo & 7742.50 & 252.90 & 287.75 & .160 \\
Grandiosidad/ autorización & 7169.00 & 251.69 & 302.84 & $\mathbf{. 0 3 9}$ \\
Insuficiente autocontrol & 7759.50 & 252.94 & 287.30 & .166 \\
Búsqueda de aprobación / & 7836.00 & 253.10 & 285.29 & .194 \\
reconocimiento & & & & \\
Negatividad/ Pesimismo & Castigo & & &
\end{tabular}


b. Se encuentra que existen diferencias estadísticamente significativas según nivel de consumo en los esquemas de desconfianza/abuso, dependencia/incompetencia, vulnerabilidad al peligro o a la enfermedad, apego confuso/yo inmaduro, autosacrificio, búsqueda de aprobación/reconocimiento, (Tabla 2).

c. Al revisar las diferencias en cuanto a los esquemas disfuncionales tempranos según nivel de consumo en cada carrera profesional; se puede observar en el caso de las carreras de salud que existen diferencias estadísticamente significativas según nivel de consumo en el esquema de sacrificio. Apreciándose además, que la media, en dicho esquema, en el caso de consumo de riesgo, es más alta en este grupo por lo cual, son los estudiantes de carreras de salud con consumo de riesgo quienes presentan este esquema en mayor medida; tal como se observa en la Tabla 3.

Tabla 3

Comparación de los esquemas disfuncionales y sus dominios según nivel de consumo de alcohol por carrera profesional

\begin{tabular}{|c|c|c|c|c|c|c|c|c|}
\hline \multirow[b]{2}{*}{ Esquema } & \multicolumn{4}{|c|}{ Ciencias de la Salud } & \multicolumn{4}{|c|}{ Ingenierías } \\
\hline & $\begin{array}{c}\text { U de } \\
\text { Mann- } \\
\text { Whitney }\end{array}$ & $\begin{array}{c}\text { Media } \\
\text { para sin } \\
\text { riesgo de } \\
\text { consumo } \\
(\mathrm{N}=291)\end{array}$ & $\begin{array}{c}\text { Media } \\
\text { para con } \\
\text { riesgo de } \\
\text { consumo } \\
(N=7)\end{array}$ & $\mathbf{p}$ & $\begin{array}{c}\text { U de } \\
\text { Mann- } \\
\text { Whitney }\end{array}$ & $\begin{array}{l}\text { Media } \\
\text { para sin } \\
\text { riesgo de } \\
\text { consumo } \\
(\mathrm{N}=181)\end{array}$ & $\begin{array}{l}\text { Media } \\
\text { para con } \\
\text { riesgo de } \\
\text { consumo } \\
(\mathrm{N}=31)\end{array}$ & $\mathbf{p}$ \\
\hline Privación Emocional & 843.00 & 10.95 & 12.00 & .434 & 2365.00 & 11.03 & 12.13 & .162 \\
\hline Abandono/ inestabilidad & 656.50 & 11.96 & 14.29 & .107 & 2336.00 & 11.53 & 12.87 & .136 \\
\hline Desconfianza/abuso & 895.00 & 12.99 & 13.43 & .583 & 2216.50 & 13.16 & 14.71 & .061 \\
\hline $\begin{array}{l}\text { Aislamiento Social/ } \\
\text { Alienación }\end{array}$ & 911.50 & 12.43 & 11.29 & .634 & 2552.00 & 12.35 & 13.32 & .421 \\
\hline Imperfección/ vergüenza & 889.50 & 9.96 & 9.29 & .565 & 2497.50 & 9.93 & 11.03 & .327 \\
\hline Fracaso & 851.50 & 10.54 & 11.43 & .457 & 2353.00 & 9.83 & 11.13 & .150 \\
\hline Depe & 888.50 & 10.41 & 10.71 & .562 & 2022.50 & 10.02 & 11.94 & .013 \\
\hline $\begin{array}{l}\text { Vulnerabilidad al peligro o a } \\
\text { la enfermedad }\end{array}$ & 900.50 & 26 & 0 & .599 & 2302.50 & 11.76 & 13.13 & .110 \\
\hline Apego confuso/ Yo inmaduro & 797.50 & 11.30 & 12.43 & .325 & 2310.00 & 11.52 & 12.84 & .115 \\
\hline Subyugación & 896.00 & 10.93 & 11.29 & .585 & 2084.50 & 10.73 & 13.06 & .022 \\
\hline Autosacrificio & 546.00 & 13.64 & 17.14 & .035 & 2338.00 & 13.60 & 14.68 & .137 \\
\hline Inhibición & 837.50 & 14.35 & 15.43 & .421 & 2779.50 & 15.31 & 15.48 & .934 \\
\hline $\begin{array}{l}\text { Normas inalcanzables / } \\
\text { hipercriticismo }\end{array}$ & 1002.50 & 16.71 & 17.14 & .943 & 2600.00 & 16.83 & 16.39 & .514 \\
\hline Grandiosidad/ autorización & 865.00 & 13.90 & 14.86 & .494 & 2678.00 & 14.78 & 15.06 & .685 \\
\hline nte autocontrol & 870.50 & 13.24 & 12.00 & .510 & 2446.50 & 14.35 & 15.39 & .254 \\
\hline $\begin{array}{l}\text { Búsqueda de aprobación / } \\
\text { reconocimiento }\end{array}$ & 750.00 & 13 & 14 & .232 & 238 & 13.73 & 14.97 & .177 \\
\hline Negatividad/ Pesimismo & 668.50 & 12.66 & 15.86 & .119 & 2783.00 & 13.92 & 14.16 & .943 \\
\hline Castigo & 965.50 & 12.62 & 12.00 & .813 & 2306.00 & 12.43 & 14.16 & .112 \\
\hline
\end{tabular}


d. En el caso de las carreras de ingenierías, se puede estimar que existen diferencias estadísticamente significativas según nivel de consumo en los esquemas de dependencia/incompetencia y subyugación. Además, en ambos casos las medias son mayores en el caso de consumo de riesgo, por lo cual son los estudiantes de ingeniería con consumo de riesgo quienes presentan ambos esquemas en mayor medida.

e. Cuando se comparan las medias en los esquemas del grupo sin consumo de riesgo tanto en estudiantes de ciencias de la salud como de ingenierías se observan que estas son bastante similares; así mismo al comparar las medias del grupo con consumo de riesgo, entre los de ciencias de la salud con los de ingenierías esta es mayor en los estudiantes de ingeniería en diez esquemas, tal como se puede observar en la Tabla 3. Por otro lado es pertinente reiterar que, a pesar de ello solo se encuentran diferencias significativas en dos esquemas. Tales resultados permiten señalar que sumados a los factores estresantes propios de la vida académica, familiar y por su etapa de vida pueden agravar su consumo o lo que es peor llegar a una dependencia en etapas de pleno desarrollo.

3. Diferencias en los esquemas disfuncionales tempranos en estudiantes universitarios de quinto ciclo, según Carrera profesional (Ciencias de la Salud e Ingenierias), de una universidad pública de la ciudad de Lima. Para el presente análisis, se ha contrastado ambos grupos, utilizando el análisis U de Mann Whitney, con el objeto de conocer si difieren en función a sus esquemas disfuncionales tempranos según carrera profesional encontrando:

a. Se encuentra al revisar los rangos promedio, un mayor puntaje en los estudiantes de ingeniería en imperfección/vergüenza, vulnerabilidad al peligro o a la enfermedad, apego confuso y búsqueda de aprobación.

b. Por otro lado cuando se analizan las diferencias en cuanto a los esquemas disfuncionales tempranos según carrera profesional, se encuentra que existen diferencias estadísticamente significativas en los esquemas de inhibición emocional, grandiosidad/ autorización, insuficiente autocontrol y negatividad/pesimismo, encontrándose que los rangos promedio son más altos en ingeniería, como se aprecia en la Tabla 4. 
Tabla 4

Comparación de los esquemas disfuncionales tempranos según carrera profesional

\begin{tabular}{lcccc}
\hline \multicolumn{1}{c}{ Esquema } & $\begin{array}{c}\text { Rango } \\
\text { U de Mann- } \\
\text { Whitney }\end{array}$ & $\begin{array}{c}\text { promedio } \\
\text { para } \\
\text { ciencias de la } \\
\text { salud }\end{array}$ & $\begin{array}{c}\text { Rango } \\
\text { promedio } \\
\text { para } \\
\text { ingenierías }\end{array}$ & p \\
\hline Privación Emocional & 30539.50 & 251.98 & 260.45 & .521 \\
Abandono/ inestabilidad & 30085.00 & 260.54 & 248.41 & .358 \\
Desconfianza/abuso & 29319.00 & 247.89 & 266.20 & .166 \\
Aislamiento Social/Alienación & 30959.50 & 253.39 & 258.46 & .701 \\
Imperfección/ vergüenza & 29887.50 & 249.79 & 263.52 & .298 \\
Fracaso & 30386.00 & 259.53 & 249.83 & .462 \\
Dependencia/ incompetencia & 31099.50 & 253.86 & 257.80 & .765 \\
Vulnerabilidad al peligro o a la enfermedad & 29149.00 & 247.32 & 267.00 & .136 \\
Apego confuso/ Yo inmaduro & 29674.00 & 249.08 & 264.53 & .242 \\
Subyugación & 30990.00 & 253.49 & 258.32 & .715 \\
Autosacrificio & 31454.50 & 255.95 & 254.87 & .935 \\
Inhibición emocional & $\mathbf{2 7 6 8 7 . 0 0}$ & $\mathbf{2 4 2 . 4 1}$ & $\mathbf{2 7 3 . 9 0}$ & $\mathbf{. 0 1 7}$ \\
Normas inalcanzables / hipercriticismo & 31286.50 & 254.49 & 256.92 & .854 \\
Grandiosidad/ autorización & $\mathbf{2 7 1 3 4 . 5 0}$ & $\mathbf{2 4 0 . 5 6}$ & $\mathbf{2 7 6 . 5 1}$ & $\mathbf{. 0 0 6}$ \\
Insuficiente autocontrol & $\mathbf{2 6 0 0 4 . 5 0}$ & $\mathbf{2 3 6 . 7 6}$ & $\mathbf{2 8 1 . 8 4}$ & $\mathbf{. 0 0 1}$ \\
Búsqueda de aprobación/reconocimiento & 29131.50 & 247.26 & 267.09 & .133 \\
Negatividad/ Pesimismo & $\mathbf{2 6 1 9 9 . 5 0}$ & $\mathbf{2 3 7 . 4 2}$ & $\mathbf{2 8 0 . 9 2}$ & $\mathbf{. 0 0 1}$ \\
Castigo & 30969.50 & 253.42 & 258.42 & .705 \\
\hline
\end{tabular}

4. Diferencias en los niveles de consumo de alcohol en estudiantes universitarios de quinto ciclo, según Carrera profesional (Ciencias de la Salud e Ingenierías) de una universidad pública de la ciudad de Lima. Igualmente que en el objetivo anterior, para el presente análisis, se ha contrastado ambos grupos, utilizando el análisis U de Mann Whitney, con el objeto de conocer si difieren en función al consumo de alcohol según carrera profesional encontrando:

- Al analizar las diferencias en cuanto al consumo de alcohol según carrera profesional, se puede apreciar que existen diferencias estadísticamente significativas (0.000) según carrera profesional en el consumo de alcohol. Además, el rango promedio es mayor en las carreras de ingeniería (285.62) en comparación con los de ciencias de la salud (234.07), por lo cual son los estudiantes de ingeniería quienes presentan mayor consumo de alcohol.

\section{Análisis Complementario}

5. Comparación entre los esquemas disfuncionales tempranos y el consumo de alcohol según género: 
- En la tabla 5 se observa las diferencias en cuanto a los esquemas disfuncionales tempranos según sexo. Así, se puede afirmar que existen diferencias estadísticamente significativas según sexo en los esquemas de privación emocional y autosacrificio. De acuerdo a los rangos promedios, los hombres presentan en mayor medida el esquema de privación emocional; y son las mujeres quienes presentan en mayor medida el esquema de autosacrificio. Se observa además que en los hombres se encuentran medias más altas en otros cinco esquemas (Imperfección /Vergüenza, Fracaso, Dependencia/Incompetencia, Subyugación y Búsqueda de aprobación) en contraste con las mujeres en un esquema más, Normas inalcanzables.

Tabla 5

Comparación de los esquemas disfuncionales tempranos según sexo

\begin{tabular}{|c|c|c|c|c|}
\hline Esquema & $\begin{array}{l}\text { U de Mann- } \\
\text { Whitney }\end{array}$ & $\begin{array}{c}\text { Rango } \\
\text { promedio para } \\
\text { los hombres }\end{array}$ & $\begin{array}{c}\text { Rango } \\
\text { promedio para } \\
\text { las mujeres }\end{array}$ & $\mathbf{P}$ \\
\hline Privación Emocional & 26659.000 & 268.83 & 234.32 & .010 \\
\hline Abandono/ inestabilidad & 30616.500 & 254.82 & 256.59 & .895 \\
\hline Desconfianza/abuso & 30199.500 & 257.52 & 252.30 & .696 \\
\hline Aislamiento Social/Alienación & 30575.000 & 254.68 & 256.80 & .874 \\
\hline Imperfección/vergüenza & 27863.000 & 264.98 & 240.44 & .066 \\
\hline Fracaso & 28466.500 & 263.05 & 243.50 & .143 \\
\hline Dependencia/incompetencia & 29648.500 & 259.28 & 249.50 & .464 \\
\hline Vulnerabilidad al peligro o a la enfermedad & 29758.500 & 258.92 & 250.06 & .507 \\
\hline Apego confuso/ Yo inmaduro & 30243.500 & 253.62 & 258.48 & .716 \\
\hline Subyugación & 28805.500 & 261.97 & 245.22 & .210 \\
\hline Autosacrificio & 26852.500 & 242.79 & 275.69 & .014 \\
\hline Inhibición emocional & 30781.500 & 255.34 & 255.75 & .976 \\
\hline Normas inalcanzables / hipercriticismo & 27706.500 & 245.52 & 271.36 & .053 \\
\hline Grandiosidad/autorización & 29896.500 & 258.48 & 250.76 & .563 \\
\hline Insuficiente autocontrol & 29778.500 & 252.14 & 260.84 & .515 \\
\hline Búsqueda de aprobación/reconocimiento & 28837.000 & 261.87 & 245.38 & .217 \\
\hline Negatividad/ Pesimismo & 30102.000 & 257.83 & 251.80 & .652 \\
\hline Castigo & 28832.000 & 261.88 & 245.36 & .216 \\
\hline
\end{tabular}


En la tabla 6 se observa las diferencias en cuanto al consumo de alcohol según sexo. Así, se puede afirmar que existen diferencias estadísticamente significativas según sexo en el consumo de alcohol. Además, el rango promedio es mayor en el caso de los hombres, por lo cual, son los hombres quienes presentan mayor consumo de alcohol.

Tabla 6

Comparación del consumo de alcohol según sexo

\begin{tabular}{ccccc}
\hline Esquema & $\begin{array}{c}\text { U de Mann- } \\
\text { Whitney }\end{array}$ & $\begin{array}{c}\text { Rango promedio } \\
\text { para los hombres }\end{array}$ & $\begin{array}{c}\text { Rango promedio } \\
\text { para las mujeres }\end{array}$ & p \\
\hline Consumo de alcohol & 23073.000 & 280.28 & 216.12 &, 000 \\
\hline
\end{tabular}

\section{Interpretación de los datos o discusión}

Una implicancia inmediata del consumo de alcohol es ubicarse en una situación de riesgo, lo cual se relaciona con la invulnerabilidad que percibe el adolescente, debido a ser una característica propia en esta etapa de vida, incrementando la posibilidad de que alumnos que consuman alcohol exterioricen conductas poniendo en riesgo su propia vida y la de los demás otros de forma más habitual. Así, el problema de consumo de alcohol va más allá de lo personal pues conlleva problemas familiares, sociales, culturales y económicos; con consecuencias nefastas a nivel intelectual, bajo rendimiento académico, problemas familiares, depresión. (Cooper, 1999 citado en Andrade, et al; 2013).).

De acuerdo con Londoño y Valencia (2010), el consumo de alcohol es percibido como un comportamiento común, como parte de la vida universitaria, básica para socializar y generadora de emociones placenteras, en escenarios donde las posibilidades y las ganancias descubiertas inducen al universitario bajo propósito de resistirse ante la exigencia de los pares, pues participa de las razones para el consumo, ahora bien puede ocurrir que no comparta dichos razonamientos, pero su baja capacidad de oposición da lugar al inicio y sostenimiento del consumo; problemática que se agudiza cuando se analiza su relación con los esquemas disfuncionales tempranos, al respecto Gantiva, et al (2010) describió una asociación entre el consumo de alcohol y los esquemas disfuncionales tempranos acentuando los de abandono, autocontrol insuficiente, desconfianza, vulnerabilidad, derecho/ grandiosidad, autosacrificio y entrampamiento. Es decir el consumo de alcohol se inscribe con estilos cognitivos determinados por sobreestimación del daño, baja tolerancia a la frustración y alta necesidad de aprobación social. Coincidentemente con Gantiva, et al, (2010) en el presente estudio en la muestra general, se encuentra correlación significativa y positiva entre autocontrol insuficiente y grandiosidad con el consumo de alcohol adicionándose los esquemas de privación emocional y búsqueda de aprobación/reconocimiento. Cuando se revisan por carrera 
profesional se encuentra relación entre el esquema castigo en los estudiantes de ciencias de la salud, indicador de ser muy exigentes consigo mismo no aceptando sus propios errores ni de los demás y, en el caso de los estudiantes de Ingenierías los esquemas que correlacionan con el consumo de alcohol son privación emocional, desconfianza/abuso y búsqueda de aprobación/reconocimiento lo que es indicador que consideran que serán humillados o se aprovecharán de ellos lo que conllevará a que no reciban afecto por lo que buscarán ganárselo a costa de perder su propia identidad.

Por otro lado, al analizar las diferencias entre los esquemas disfuncionales tempranos con los niveles de consumo de alcohol en la muestra total de estudiantes universitarios de quinto ciclo se encuentran mayores rangos medios en la presencia de esquemas disfuncionales en el grupo con riesgo para el consumo que en el de sin riesgo. Es así que desde esta aspecto los esquemas cognitivos influirán de manera concreta en la forma en que los sujetos perciben el mundo y, como consecuencia, esta distorsión cognitiva llevará al sujeto a padecer desadaptación o disfuncionalidad ante situaciones que no tendrían que ser así, ya que no se corresponden con la realidad (Young, 1999), es decir que pueden ser disparadores de comportamientos de riesgo entre ello el incremento del consumo de alcohol.

Al revisar la muestra general se encuentra que solo el $8 \%$ han reportado consumo de alcohol de riesgo, lo cual amerita dos posturas, una de ellas proponer programas para que no lleguen a consumo de riesgo y la otra, realizar investigaciones de mayor especificidad para medir los niveles de consumo ello debido a que cuando se analizan los esquemas se encuentran diferencias estadísticamente significativas en los esquemas de desconfianza abuso, dependencia/incompetencia, vulnerabilidad al peligro o a la enfermedad, apego confuso, autosacrificio y búsqueda de aprobación/reconocimiento. Resultados que coinciden con Chattás y Góngora, (2008) quien señala que en los sujetos sin adicción se destacan los esquemas de Privación Emocional, Abandono / Inestabilidad; Desconfianza / Abuso y Dependencia / Incompetencia y que los sujetos con adicciones poseen esquemas disfuncionales tempranos más severos. Asimismo Gantiva, et al (2010) también encuentra diferencias significativas en los esquemas de abandono, autocontrol insuficiente, desconfianza, vulnerabilidad, autosacrificio, inhibición emocional, derecho/grandiosidad y apego confuso.

En lo referente a las diferencias entre los esquemas disfuncionales tempranos según nivel de consumo de alcohol en cada carrera profesional se encuentra que en ciencias de la salud existe diferencias significativas en el esquema sacrificio en el grupo de consumo de riesgo, ahora bien en el de Ingenierías existen diferencias significativas en el grupo de riesgo, en los esquemas de dependencia/ incompetencia y subyugación, siendo las medias mayores en el caso del grupo con consumo de riesgo; datos coincidentes con los de Montaño, Morales y Gómez (2011) en cuanto al nivel de riesgo encuentra que la facultad de ingeniería (Bogotá) 
presenta el mayor porcentaje (41\%), en el nivel perjudicial y dependiente, asimismo encuentra que la facultad de psicología, una de las carreras de ciencias de la salud (denominada así en la universidad pública del estudio) presenta un porcentaje menor $(11,8 \%)$.

Cuando se analizan las diferencias en relación a esquemas disfuncionales tempranos según carrera profesional se encuentra diferencias significativas en los esquemas inhibición emocional, grandiosidad, insuficiente autocontrol y negatividad pesimismo y con mayor rango medio en la carrera de ingeniería. Se hace relevante destacar que los esquemas disfuncionales tempranos intervienen en el consumo de alcohol, influyendo en la modo de portarse, de pensar y asumir decisiones sobre eventos que pasan en la vida, asimismo influyen en las maneras que asumimos a las personas y en la forma de establecer vínculos sociales, (Hederich \& Camargo, 2001; citado en Gantiva, et al, 2010)). En este sentido señalan Andrade, et al (2013), que el hecho de tener pares con problemas socio familiares y pares consumidores, es uno de los factores de riesgo asociado al consumo de la mayoría de las sustancias psicoactivas.

De acuerdo con Londoño y Valencia, (2010), el consumo de alcohol se aprecia como una conducta cotidiana del universitario, agregan además, que es incuestionable la relación entre la influencia de grupo y el consumo de alcohol, puesto que los jóvenes en esta etapa son altamente influenciables. Así de acuerdo con los porcentajes de consumo de alcohol reportados en el Perú, como en otros países (España, Estados Unidos, México y Colombia) se reconoce que el consumo de alcohol es un fenómeno que tiene alta prevalencia y que cada vez inicia a edades más tempranas; en el Perú en promedio los 13 años de edad. Finalmente en el presente estudio se encuentra claras diferencias significativas según carrera profesional y mayor rango medio en las carreras de ingeniería e inclusive es en este grupo que existen mayor número de estudiantes con consumo de riesgo que en los de ciencias de la salud. Ello se agrava pues los esquemas asociados al consumo de riesgo a tener problemas de autoestima, conflictos o violencia familiar, estrés académico, falta de habilidades sociales, entre otros pueden generar dependencia al consumo de alcohol, riesgo para la salud individual, afectando de igual forma la salud pública (Cicua, Méndez, Muñoz, 2008; citado en Andrade, et al 2013). A ello se suma lo encontrado por Malacas (2013) señalando que los esquemas disfuncionales tempranos que se relacionaron más fuertemente y singularmente con el rasgo de agresividad y síntomas depresivos, fueron Desconfianza, Grandiosidad y Autocontrol Insuficiente que son los mismos esquemas que encontramos en el presente estudio.

Al contrastar los esquemas disfuncionales y el consumo de alcohol según sexo, se aprecia la presencia de más esquemas disfuncionales en los hombres (Autosacrificio, Imperfección /Vergüenza, Fracaso, Dependencia/Incompetencia, Subyugación y Búsqueda de aprobación) en contraste con las mujeres, dos 
esquemas autosacrificio y Normas inalcanzables. Asimismo, son los hombres los que presentan mayor consumo de alcohol.

\section{CONCLUSIONES}

En la muestra total constituida por estudiantes universitarios de las carreras de ciencias de la salud e ingeniería existe una relación positiva y significativa entre los esquemas de privación emocional, grandiosidad/autorización, insuficiente autocontrol y búsqueda de aprobación/ reconocimiento con el consumo de alcohol.

Los universitarios de ciencias de la salud obtiene una correlación significativa positiva entre el esquema castigo con el consumo de alcohol. Este grupo considera que las personas deben ser castigados por sus errores, lo que conlleva a impacienta y a no tolerar sus propios errores ni la de los demás; en el caso de los estudiantes de las carreras de ingeniería, también se encuentra correlación significativa positiva entre los esquemas de privación emocional, desconfianza/abuso y búsqueda de aprobación/reconocimiento con el consumo de alcohol. A diferencia del grupo anterior, estos estudiantes tienden a considerar que los demás los pueden humillar o aprovecharse de él, que conllevará a que traten de ganarse el afecto de los demás a costa de su propia identidad.

Al contrastar la muestra total de los estudiantes universitarios de las carreras de ciencias de la salud e ingenierías divididos en consumo de riesgo y sin riesgo se encuentran diferencias en los esquemas según nivel de consumo, presentado mayores rangos promedio en el grupo con riesgo. Encontrándose diferencias significativas según nivel de consumo en los esquemas de desconfianza/ abuso, apego confuso, autosacrificio, reconocimiento.

En las carreras de ciencias de la salud en el grupo de riesgo predomina el esquema autosacrificio.

En las carreras de ingeniería se encuentra medias mayores en el grupo con consumo de riesgo en los esquemas dependencia/incompetencia y subyugación,

Existe mayor número de estudiantes de las carreras de Ingeniería con consumo de riesgo (31) en comparación con los de ciencias de la salud (7). Son los estudiantes de ingeniería los que presentan mayor consumo de alcohol.

Se encuentra, al comparar las medias del grupo con consumo de riesgo, que esta es mayor en la carrera de ingeniería en diez esquemas.

Los rangos promedio son más altos y existen diferencias estadísticamente significativa en ingeniería, al comparar los esquemas según carrera profesional en los esquemas de inhibición emocional, grandiosidad/ autorización, insuficiente autocontrol y negatividad/pesimismo. 
Existen más esquemas disfuncionales tempranos en los hombres que en las mujeres y presentan mayor consumo de alcohol los universitarios que las universitarias.

\section{REFERENCIAS BIBLIOGRÁFICAS.}

Andrade, J., Yepes, Á. y Sabogal, M. (2013). Resistencia a la presión de grupo y consumo de alcohol en 50 jóvenes universitarios entre los 16 y 22 años de la Universidad de San Buenaventura Medellín extensión Ibagué. Revista Colombiana de Ciencias Sociales, 4(1), 11-22.

Babor, T.; Higgins, J.; Saunders, J. \& Monteiro, M. (2001). Cuestionario de Identificación de los Transtornos debidos al Consumo de Alcohol (AUDIT). Organización Mundial de la Salud. Generalitat Valenzania. www.who.int/substance_abuse/activities/en/ AUDITmanualSpanish.

Chattás, A. y Góngora, V. (2008) "Esquemas desadaptativos tempranos en pacientes con trastorno por abuso de sustancias". Acta PsiquiátPsicol Am Lat., 54(2): 87-95.

Castrillón, D; Chaves, L.; Ferrer, A.; Londoño, N.; Maestre, K. y Marín, C. (2005) Validación del YongSchemaQuestionnaire Long Form - SecondEdition (YSQ - L2) en población colombiana. Revista Latinoamericana Psicología. 37(.3). Bogotá. Disponible en pepsic.bvsalud.org/scielo.php?script=sci_arttext\&pid=S0120

Comunidad Andina (2013). II Estudio epidemiológico andino sobre consumo de drogas en la población universitaria. Informe Perú, 2012. CAN-Proyecto PRADICAN. Unión Europea. Lima.

Comisión Nacional para el Desarrollo y Vida sin Drogas - DEVIDA (2013). IV Estudio Nacional Prevención y Consumo de Drogas en Estudiantes de Secundaria 2012. Lima: DEVIDA.

Comisión Nacional para el Desarrollo y Vida sin Drogas - DEVIDA (2014). I Estudio sobre prevención y consumo de drogas en la población general de Lima Metropolitana y el Callao - 2013. Lima: DEVIDA - Observatorio Peruano de Drogas.

Gantiva, C; Bello, J; Vanegas, E y Sastoque, Y. (2010) "Relación entre el consumo excesivo de alcohol y esquemas maladaptativos tempranos en estudiantes universitarios". Revista Colombiana de Psiquiatría, 39, 362-374.

Hernández, R.; Fernández, C. y Baptista, P. (2010). Metodología de la Investigación. Mexico: McGra-Hill.

Londoño C., García, W, Valencia, S. y Vinaccia, S. (2005). Expectativas frente al consumo de alcohol en jóvenes universitarios colombianos, Anales de psicología, 21(2), 259-267. Medellín Colombia: Universidad Católica de Colombia, Universidad San Buenaventura - Medellín.

Londoño, C., Valencia, C. (2010). Resistencia de la presión de grupo, creencias acerca del consumo y consumo de alcohol en universitarios. Anales de Psicología. 26(1) 27-33.

McGinn, L. K., \& Young, J. E. (1996). Terapia basada en esquemas. In P. M. Salkauskis (Ed.), Frontiers of cognitive therapy (pp. 182-207). New York: Guilford Press. 
Malacas, C. (2013). Esquemas Disfuncionales Tempranos en Consumidores de Sustancias Psicoactivas Internados en Comunidades Terapéuticas. Lima. Recuperado en www. derecho.usmp.edu.pe/sapere/ediciones/...8/.../Esquemas_dis_Paula\%20Gaido.pd

Montaño, M.; Morales, A. y Gómez, M. (2011). Consumo de alcohol en estudiantes de la Universidad de San Buenaventura sede Bogotá. Tesis para optar el grado de Psicólogo. Universidad de San Buenaventura. Bogotá-Colombia. Recuperado en bibliotecadigital.usb.edu.co/.../1/Consumo_alcohol_estudiantes_Montaño_2011.pdf

Young, J. (1999). Cognitive therapy for personality disorders: A schema-focused approach. Sarasota: Professional Resource Press. 